\title{
SCHRIFTEN
}

ZUR GESCHICHTE UND KULTUR 8 DES ALTEN ORIENTS

BERLINER TURFANTEXTE IV

AKADEMIE

DER WISSENSCHAFTEN DER DDR ZENTRALINSTITUT FUR ALTE GESCHICHTE UND ARCHAXOLOGIE

WERNER SUNDERMANN

\section{Mittelpersische und} parthische kosmogonische und Parabeltexte der Manichäer mit einigen Bemerkungen zu Motiven der Parabeltexte von FRIEDMAR GEISSLER

Mit 156 Faksimiles auf 53 Tafeln 
Redaktion: Friedmar Geißler

Ersohienen im Akademie-Verlag, 108 Berlin, Leipziger Straße 3-4

Copyright 1973 by Akademie-Verlag Berlin

Lizenznummer : $202 \cdot 100 / 116 / 73$

Herstellung: IV/2/14 VEB Druckerei ^Gottfried Wilhelm Leibniz», 445 Gräfenhainichen/DDR 3880

Bestellnummer: $7522164(2146 / 8)$. LSV 0876

Printed in GDR

EVP 54,- 